\title{
Analysis and editing the maize gamete interactions and fusion genes
}

\author{
Moiseeva E.M., Gusev Yu.S., Fadeev V.V., Mazilov S.I., Chumakov M.I.* \\ Institute of Biochemistry and Physiology of Plants and Microorganisms, Russian Academy of Sciences, \\ Saratov, Russia \\ *email: chumakov_m@ibppm.ru
}

The molecular-genetic aspects of double fertilization in angiosperms plants, discovered by S. Navashin over a hundred years ago (1896), still remains poorly understood. In the case of gynogenesis one of the spermium does not merge with the egg cell and haploid plant develop the parthenogenetic embryo from unfertilized egg cell. The knowledge of the mechanism of gamete membrane interaction and fusion may be of practical use for haploid-inducing maize line production. We analyzed the gynogenesis (parthenogenesis) genes in maize, in they involvement in the gamete membrane interaction and fusion. Particular, the maize $Z m \_g e x 2$ and $Z m \_g c s 1$ genes, which we for the first time described in 2017 may be involved in gynogenesis also. For creation the CRISPR/Cas 9 constructs on the base pRGEB-32BAR vector, designed for maize plants, were synthesized three gRNA for Zm_gex2 and two gRNA for Zm_gcs 1 maize genes, and introduced into the maize genome for knockout of these genes by the in planta method. We harvested about four thousand seeds after agrobacterial transformation BM maize line with CRISPR/Cas9-constructs with sgRNA to Zm_gex2 and Zm_gcs1 genes by the in planta method. PCR was applied for analysis of two thousand 2-week old seedlings, and $15 \mathrm{~T}$ DNA insertions was found, but only for 7 maize plants transformation was confirmed by the sequences method.

Acknowledgements: This work was carried out within the Russian Program of Basic Research of the state academies of sciences for 2021-2023 (registration No. 121031700141-7) and supported by the Russian Foundation for Basic Research, grants №20-016-00020a (Zm_gex2) and No. 20-316-80020/20 (Zm_gcsl). 